\title{
Music therapy for in-patients with schizophrenia
}

\author{
Exploratory randomised controlled trial
}

\author{
NAKUL TALWAR, MIKE J. CR AWFORD, ANNA MARATOS, ULA NUR, \\ ORII MCDERMOTT and SIMON PROCTER
}

\section{Background Music therapy may provide a means of improving mental health among people with schizophrenia, but its effects in acute psychoses have not been explored.}

Aims To examine the feasibility of a randomised trial of music therapy for inpatients with schizophrenia, and explore its effects on mental health.

Method Up to 12 weeks of individual music therapy plus standard care were compared with standard care alone. Masked assessments of mental health, global functioning and satisfaction with care were conducted at 3 months.

Results Of 115 eligible patients 81 (70\%) were randomised. Two-thirds of those randomised to music therapy attended at least four sessions (median attendance, eight sessions). Multivariate analysis demonstrated a trend towards improved symptom scores among those randomised to music therapy, especially in general symptoms of schizophrenia.

Conclusions A randomised trial of music therapy for in-patients with schizophrenia is feasible. The effects and cost-effectiveness of music therapy for acute psychosis should be further explored in an explanatory randomised trial.

Declaration of interest None.
Despite the development of communitybased services which reduce the need for in-patient care, many people with severe mental illness continue to need periods of in-patient treatment. Over recent years, concerns have been expressed about the quality of in-patient care: in particular the lack of therapeutic contact between patients and staff (Sainsbury Centre for Mental Health, 1998; Department of Health, 2003). Music therapy is a form of psychological treatment which may be able to engage people with severe mental illness (Pavlicevic \& Trevarthen, 1989). Whereas the effects of listening to music and singing have been examined among in-patients with schizophrenia (Tang et al, 1994; Hayashi et al, 2002), co-improvisational music therapy, the form generally practised in in-patient settings in Western Europe, has not been evaluated (Bruscia, 1998). We therefore conducted an exploratory randomised trial of music therapy for in patients with schizophrenia in order to examine the feasibility of a trial and to estimate the impact of this intervention on mental health, global functioning and satisfaction with care.

\section{METHOD}

\section{Participants}

We conducted a multi-centre, parallel-arm, randomised controlled trial, with baseline and follow-up measures assessed at 12 weeks. The study sample was recruited from in-patients at one of four hospitals in central and inner London (Park Royal Centre for Mental Health, Paterson Centre, St Charles' Hospital and St Clement's Hospital). These hospitals serve a combined population of approximately 400000 residents who are on average younger, more mobile and more ethnically diverse than in other parts of England and Wales (Office for National Statistics, 2003).

The study population were in-patients aged over 18 years with a primary diagnosis of schizophrenia, or schizophrenia-like psychoses (ICD-10 (World Health Organization, 1992): F20-F29). Patients being treated on a compulsory basis or lacking capacity were included so long as they provided assent and those involved in their care were happy for them to participate. Those with a secondary diagnosis of organic psychosis or dementia and those who spoke insufficient English to complete the baseline interview without the help of an interpreter were excluded from the study. Patients involved in the trial were excluded from music and other arts therapies (art, dance and movement, and drama therapy) during the trial. Local research ethics committee approval was obtained before the start of data collection.

\section{Experimental and controlled treatment}

All study patients received routine standard care including nursing care and access to a range of occupational, social and other activities provided as part of the in-patient programme. In addition, those randomised to music therapy received up to 12 individual sessions of music therapy. In keeping with clinical practice for treating people with acute psychosis, we evaluated the impact of individual music therapy in this trial. Patients who were discharged from the ward before the end of 12 weeks were encouraged to continue attending music therapy on an out-patient basis for the remainder of this period. Five music therapists took part in the trial. All had trained on courses approved by the Health Professions Council, and received fortnightly supervision from a senior music therapist (A.M.) throughout the study period.

Therapy sessions took place once a week, for up to $45 \mathrm{~min}$. During sessions, patients were given access to a range of musical instruments and encouraged to use these to express themselves (Ansdell, 1995). As per routine practice, all sessions were digitally recorded. The focus of the therapy was on co-creating improvised music, with talking used to guide, interpret or enhance the musical experience. Initially the therapist listens carefully to the patient's music and accompanies them closely, seeking to meet their emotional state in musical terms. Subsequently the therapist offers interventions in the form of opportunities to extend or vary the nature of the musical interaction (Pavlicevic 
et al, 1994; Bruscia, 1998). Supervision of music therapists involves reflection on the meaning of the interaction in an interpersonal context, and close examination of the co-improvisations by listening back to recordings of the sessions (Turry, 1998).

A random sample of these recordings was examined at the end of the trial in order to assess treatment fidelity. This involved listening to the recording and quantifying the amount of time spent by patients and therapists co-improvising music, playing solo, communicating verbally or in silence.

Those randomised to routine care alone were placed on a waiting list and offered music therapy at the end of the trial period.

\section{Outcome measures}

Our primary outcome measure was the total score on the Positive and Negative Syndrome Scale (PANSS; Kay et al, 1987), a 30 -item rating scale which has been widely used to examine changes in symptoms among people with schizophrenia and other psychotic illnesses. Our secondary outcomes were selected on the basis of their wide use in studies of psychosocial interventions for people with schizophrenia. They comprised changes in the positive, negative and general sub-scales of the PANSS; global functioning, assessed using the Global Assessment of Functioning Scale (Jones et al, 1995); and satisfaction with care, measured by the Client Satisfaction Questionnaire (Atkinson \& Greenfield, 1994).

Data on all outcome measures were collected before randomisation and 3 months later. In addition, baseline demographic data, clinical details and details of all medication were collected from patient interview and in-patient notes.

\section{Procedures}

In consultation with ward staff, patients who met study criteria were approached, provided with written and verbal information about the study and asked whether they would be willing to take part in the trial. Those willing to participate were asked to provide written informed consent or assent (Medical Research Council, 1998). Those meeting study inclusion and not exclusion criteria completed baseline assessment and were then assessed by a local music therapist for suitability for music therapy. Those judged suitable were then randomised to therapy plus routine care or to routine care alone, by block randomisation stratified for hospital site, using randomisation lists derived from a computer program. A randomisation ratio of therapy to routine care of 2:3 was used in order to balance researcher time and the availability of music sessions.

All follow-up interviews were conducted by a researcher masked to treatment condition (N.T.) 3 months after randomisation. Patients who were not followed up within 1 month after this date were considered lost to follow-up. Extensive steps were taken to mask the researcher to the participant's allocation status. Randomisation was conducted by a person independent of the researcher, and therapists and patients were instructed not to talk to the researcher about which arm of the trial they were in. All participants were offered a $£ 10$ postal order following completion of the 3-month follow-up interview.

\section{Sample size and data analysis}

In the absence of previous research providing an estimate of changes in our primary outcome (total PANSS score at follow-up), we set out to recruit a sample of a similar order to the 76 people that Tang and colleagues involved when they demonstrated statistically significant reductions in negative symptoms of schizophrenia among long-stay patients who received sessions in which they listened to music and took part in group singing (Tang et al, 1994).

Data from patient notes and interviews were double-entered into an Excel database and transferred to a STATA file (version 8.0) for data analysis. Multiple imputation was used to account for the missing data in outcome measures at follow-up. This method imputes $m>1$ plausible values for each missing value, under the assumption of missing at random. The missing at random holds when missing data are different from the observed data, but the pattern of missing data is traceable from the observed data (Rubin, 1987). Results are then combined using multiple imputation rules.

Baseline data including diagnosis and other routine data were used to ascertain whether study groups differed. The distribution of changes in mean PANSS scores 3 months after randomisation among the two groups was examined. Univariate tests examined differences in total PANSS scores between those randomised to experimental or control treatment on an intention-totreat basis. Regression analysis adjusted for any differences in potential confounding factors. Multivariate models were built by forward stepwise regression.

\section{RESULTS}

During the study period 123 people were screened, of whom 113 (92\%) were eligible to participate in the study (Fig. 1); 31 eligible patients $(27 \%)$ refused to take part in the study and $1(1 \%)$ was considered unsuitable for music therapy following assessment by a music therapist. The remaining 81 ( $72 \%$ of eligible patients) were randomised; 60 (74\%) were men, and ages ranged from 18 to 64 years (mean 37). Of the 81 participants, $33(41 \%)$ were randomised to music therapy and 48 (59\%) to control treatment. Characteristics of those randomised to each arm of the trial are presented in Table 1.

At 3-month follow-up, 69 interviews $(85 \%)$ were completed. The rates of follow-up were $85 \%$ in both arms of the trial. We are aware of only one occasion when a breach of the study protocol led the researcher to become unmasked. As a further test of masking, N.T. attempted to guess the allocation status of each of the participants after 3-month follow-up data had been collected. The level of agreement beyond chance was in the low range (kappa $=0.31, P<0.01$ ).

Examination of a random sample of recordings of $810 \mathrm{~min}$ of music therapy from 21 sessions revealed that $648 \mathrm{~min}$ $(80 \%)$ were spent in musical coimprovisation; $118 \mathrm{~min}(14.5 \%)$ in verbal dialogue; $16 \mathrm{~min}(2 \%)$ with the therapist and other patients singing or playing pre-composed music together; $13 \mathrm{~min}(1.6 \%)$ in silence; and $11 \mathrm{~min}$ $(1.4 \%)$ with the patient singing or playing unaccompanied.

Study outcomes among those in each arm of the trial are compared in Table 2 . Change in total PANSS scores among those in the therapy arm of the trial were significantly greater than those in the standard care arm of the trial. Modest differences in secondary outcomes did not reach statistical significance. Univariate analysis suggested that two other variables, baseline PANSS score and gender, were associated with differences in symptom scores at 3 months. These two factors were therefore included in a multivariate model examining factors associated with reductions in 


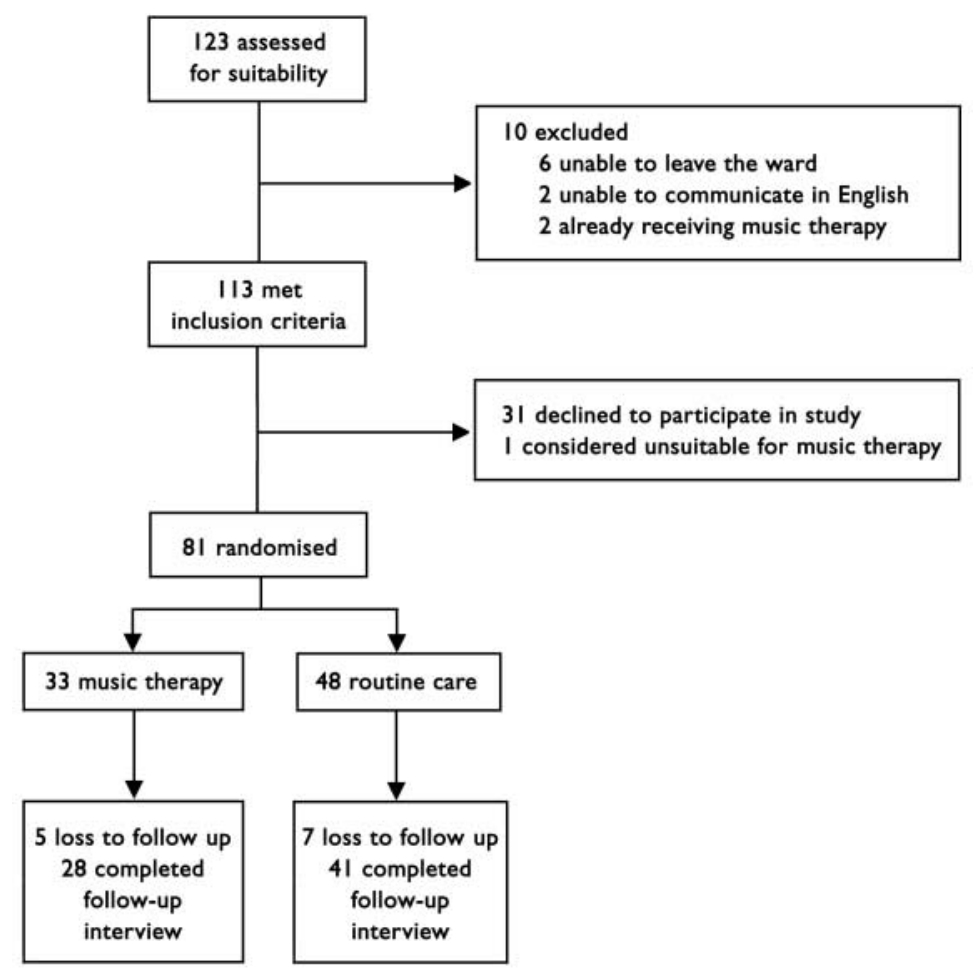

Fig. I. CONSORT diagram showing patient flow through the study (from screening to 3-month follow-up).

symptom scores (and are presented in Table 3).

Of those randomised to music, all attended at least one session and $7(21 \%)$ attended all 12 sessions. The median attendance was eight sessions and 22 people $(67 \%)$ attended at least four sessions. The most frequently stated reason for ending therapy was that the patient was discharged from the ward. When discharge took place after one or two sessions of music therapy re-attendance was rare, but when patients had already attended several sessions as in-patients, they generally returned to complete their sessions following discharge from hospital.

\section{DISCUSSION}

To our knowledge this is the first randomised trial of music therapy for people with acute psychosis. The study demonstrated that such a trial is feasible, and that the majority of patients who are offered music therapy will accept it. Over half the study sample were being treated on a compulsory basis and had not attended any in-patient activity in the 2 weeks before randomisation yet, on average, those randomised to music therapy subsequently attended seven sessions of therapy. Referral for music therapy was associated with short-term reductions in general and negative symptoms of schizophrenia, although differences in baseline characteristics of the sample may have been responsible for these apparent differences.

\section{Limitations}

Limited time and resources meant that we were only able to randomise 81 people. Although this provided a sufficiently large sample to enable us to estimate the scale of impact of music therapy, it was insufficient to identify statistically significant differences in treatment outcomes between study groups. Differences in baseline characteristics of patients at the start of the trial further reduced the explanatory power of the study. We stratified the sample by study site, but minimisation would have enabled us to ensure that baseline characteristics in each arm of the trial were better balanced (Altman \& Bland, 2005). Masking of researchers in trials of complex interventions is always a challenging task. We are aware of only one occasion when allocation status was revealed before completion of assessment of patient outcomes. However, we cannot rule out the possibility that a degree of unmasking affected the assessment of study outcomes.

\section{Changes in symptom scores}

Differences in symptom scores at the end of treatment were smaller than those reported in previous studies of music therapy for inpatients (Tang et al, 1994; Hayashi et al, 2002). Multiple factors could be responsible for these differences. First, previous studies provided more intensive interventions and achieved higher levels of attendance at therapy sessions. For instance, Tang and colleagues reported that all patients involved in their trial attended all music therapy sessions. We were keen to examine the effects of music therapy in an acute in-patient setting. The length of in-patient stay has decreased in most mental health units over recent years (National Statistics, 2004), and in the present study the majority of participants had left the in-patient unit before the end of therapy. As a result of this, a third of those randomised to music therapy had fewer than the four sessions we aimed to deliver to them. Previous studies have examined the impact of music therapy among people with chronic schizophrenia, who generally have the more negative symptoms. It is interesting that in this study we saw the greatest differences in general and negative symptoms, and it is possible that music therapy has particular effects on these symptoms, effects which are likely to be most apparent when the intervention is used among people with chronic schizophrenia.

General symptoms of schizophrenia measured by the PANSS refer to disturbances in depressive cognitions and depressed mood. Psychotropic medication has limited effects on these symptoms (Siris, 2000), but they are the ones most strongly associated with patient judgements about the value of the treatment they receive (van Os et al, 1999). Further consideration needs to be given to the potential that adjunctive music therapy has for improving such symptoms among people with schizophrenia.

We found little difference in other secondary outcomes measured in the trial. This may be because music therapy does not have an effect on these outcomes, but it could also be the result of the limited statistical power of a study of this size. An alternative explanation is that we did not follow patients up long enough for changes 
Table I Baseline characteristics of 81 patients randomised to music therapy or routine care

\begin{tabular}{|c|c|c|c|c|}
\hline \multirow{2}{*}{$\begin{array}{l}\text { Characteristic } \\
\text { Mean age, years (s.d.) }\end{array}$} & \multicolumn{2}{|c|}{$\begin{array}{c}\text { Music therapy } \\
n=33(\%)\end{array}$} & \multicolumn{2}{|c|}{$\begin{array}{c}\text { Routine care } \\
n=48(\%)\end{array}$} \\
\hline & 35.4 & $(10.6)$ & 38.7 & (II.7) \\
\hline Male gender & 23 & $(69.7)$ & 37 & (77.I) \\
\hline Married & 4 & $(12.1)$ & 6 & $(12.5)$ \\
\hline White British & 10 & (30.3) & 25 & (5I.I) \\
\hline No academic qualifications & II & (33.3) & 12 & $(25.0)$ \\
\hline \multicolumn{5}{|l|}{ Diagnosis } \\
\hline Schizophrenia & 10 & $(30.3)$ & 13 & $(27.1)$ \\
\hline Paranoid schizophrenia & 10 & (30.3) & 23 & (47.9) \\
\hline Chronic schizophrenia & 4 & $(12.1)$ & 5 & $(10.4)$ \\
\hline Other & 9 & (27.2) & 8 & $(16.7)$ \\
\hline Mental Health Act 1983 status: compulsory & 18 & (54.5) & 29 & (58.3) \\
\hline Median number of groups attended during the previous & 0 & & I & \\
\hline \multicolumn{5}{|l|}{2 weeks } \\
\hline Medication: CPZ equivalents, mg (s.d.) & \multicolumn{2}{|c|}{$417.8(340.8)$} & \multicolumn{2}{|c|}{$478.5(396.5)$} \\
\hline PANSS - total score (s.d.) & 73.1 & $(13.4)$ & 70.8 & $(12.8)$ \\
\hline GAF score (s.d.) & 54.2 & $(I I .4)$ & 55.7 & $(9.8)$ \\
\hline Satisfaction score (s.d.) & 20.2 & (5.6) & 20.2 & $(4.1)$ \\
\hline
\end{tabular}

CPZ, chlorpromazine; PANSS, Positive and Negative Syndrome Scale; GAF, Global Assessment of Functioning.

Table 2 Changes in primary and secondary outcomes among those in the experimental and control arms at baseline and at 3 months

\begin{tabular}{|c|c|c|c|c|}
\hline Symptom scores & $\begin{array}{c}\text { Baseline } \\
(n=8 I)\end{array}$ & $\begin{array}{l}12 \text { weeks follow-up } \\
\quad(n=8 \mid)\end{array}$ & $\begin{array}{l}\text { Change in } \\
\text { scores }\end{array}$ & $\begin{array}{c}\text { Difference } \\
\text { (t-test) }\end{array}$ \\
\hline \multicolumn{5}{|l|}{ PANSS - total } \\
\hline Standard care & 70.77 (12.82) & $67.81 \quad(14.56)$ & 2.96 & \multirow{2}{*}{$6.04(2.04)^{*}$} \\
\hline Music therapy & 73.09 (13.4I) & 64.09 (13.78) & 9.00 & \\
\hline \multicolumn{5}{|l|}{ PANSS - general } \\
\hline Standard care & $35.43 \quad(7.01)$ & 34.54 (7.09) & 0.89 & \multirow{2}{*}{3.97 (1.69) } \\
\hline Music therapy & $37.21 \quad(7.09)$ & $32.35 \quad(6.57)$ & 4.86 & \\
\hline \multicolumn{5}{|l|}{ PANSS - positive } \\
\hline Standard care & $16.52(3.57)$ & $14.57 \quad(3.41)$ & 1.95 & \multirow{2}{*}{$0.56(0.78)$} \\
\hline Music therapy & 16.12 & $13.61 \quad(3.42)$ & 2.51 & \\
\hline \multicolumn{5}{|l|}{ PANSS - negative } \\
\hline Standard care & I8.8I (4.72) & $18.51 \quad(5.00)$ & 0.30 & \multirow{2}{*}{$2.42(1.32)$} \\
\hline Music therapy & 19.76 & $17.04(4.81)$ & 2.72 & \\
\hline \multicolumn{5}{|l|}{ Satisfaction } \\
\hline Standard care & $20.18 \quad(4.07)$ & $20.51 \quad(4.19)$ & 0.33 & \multirow{2}{*}{$1.49(0.99)$} \\
\hline Music therapy & $20.18 \quad(5.60)$ & $22.00(5.05)$ & 1.82 & \\
\hline \multicolumn{5}{|l|}{ Global functioning } \\
\hline Standard care & $55.65(9.81)$ & $60.25(9.27)$ & 4.60 & \multirow{2}{*}{$0.14(0.69)$} \\
\hline Music therapy & 54.18 (II.39) & $58.92(10.90)$ & 4.74 & \\
\hline
\end{tabular}

PANSS, Positive and Negative Syndrome Scale.

$* P=0.045$.

Table 3 Factors associated with differences in total PANSS scores at 3 months among $8 \mathrm{I}$ study participants

\begin{tabular}{lcrr}
\hline Variable & $\begin{array}{c}\text { Coefficient } \\
(95 \% \text { confidence limits })\end{array}$ & Standard error & $P$ \\
\hline Baseline PANSS score & $0.28(0.05$ to 0.49$)$ & 0.11 & 0.02 \\
Male gender & $10.44(3.85$ to I7.03) & 3.11 & $<0.01$ \\
Randomised status - music therapy & $-4.52(-1 \mathrm{Il} .30$ to I.2I) & 3.19 & 0.11 \\
\hline
\end{tabular}

PANSS, Positive and Negative Syndrome Scale. to become apparent. A lag between impact on symptom scores and changes in social functioning has been reported in previous trials examining psychosocial interventions for people with schizophrenia (Kemp et al, 1996).

\section{Future research}

We believe that findings from this study provide sufficient evidence to justify a larger explanatory trial of music therapy for people with schizophrenia. We estimate that data on 214 people would need to be obtained in order to have $80 \%$ power to explore a difference of the magnitude we found at a $5 \%$ level of statistical significance. Recruitment of participants from a range of acute and less acute settings would provide an opportunity to see whether music therapy has differential effects on different symptom groupings. Such a trial would benefit from a longer follow-up period to examine whether the impact of therapy is sustained. It should also include more detailed measures of mood which may be particularly responsive to this form of intervention. A larger trial could also provide an opportunity to examine the active ingredients of music therapy for people with schizophrenia. This could be achieved either through using an active control group to account for non-specific aspects of therapy such as time spent with a therapist, or by combining the collection and analysis of qualitative and quantitative data in order to examine the relationship between the process and outcomes of music therapy.

\section{ACKNOWLEDGEMENTS}

We are grateful to study participants, to Claire Threlfall and Sarah Wilson for delivering music therapy and for the support of Central and North West London, and East London and The City, NHS Mental Health Trusts. N.T. was funded through a Priory training grant.

\section{REFERENCES}

Altman, D. G. \& Bland, J. M. (2005) Treatment allocation by minimisation. BMJ, 330, 843 .

Ansdell, G. (1995) Music for Life. London: Jessica Kingsley.

Atkinson, C. \& Greenfield, T. (1994) Client Satisfaction Questionnaire-8 and Service Satisfaction Scale 30. In Psychological Testing: Treatment Planning and Outcome Assessment. San Francisco, CA: Lawrence Erlbaum.

Bruscia, K. E. (1998) Defining Music Therapy. Gilsum, $\mathrm{NH}$ : Barcelona. 
Department of Health (2003) Mental Health Policy Implementation Guide: Adult Acute In-Patient Care Provision. London: Department of Health.

Hayashi, N., Tanabe, Y., Nakagawa, S., et al (2002) Effects of group musical therapy on inpatients with chronic psychoses: a controlled study. Psychiatry and Clinical Neurosciences, 56, 187-193.

Jones, S. H., Thornicroft, G., Coffey, M., et al (1995) A brief mental health outcome scale. Reliability and validity of the Global Assessment of Functioning (GAF). British Journal of Psychiatry, 166, 654-659.

Kay, S. R., Fiszbein, A. \& Opler, L. A. (1987) The positive and negative syndrome scale (PANSS) for schizophrenia. Schizophrenia Bulletin, 13, 26I-276.

Kemp, R., Hayward, P., Appplewhaite, G., et al (1996) Compliance therapy in psychotic patients: randomised controlled trial. BMJ, 3/2, 345-349.

Medical Research Council (1998) MRC Guidelines for Good Clinical Practice in Clinical Trials. London: Medical Research Council.

National Statistics (2004) NHS In-Patient Activity for Sick and Disabled People: Social Trends 34. London: Office for National Statistics.

Office for National Statistics (2003) Census 2001: National Report for England and Wales. London: Office for National Statistics.

Pavlicevic, M. \& Trevarthen, C. (1989) A musical assessment of psychiatric states in adults. Psychopathology, 22, 325-334.

NAKUL TALWAR, MRCPsych, Central and North West London Mental Health NHS Trust, UK; MIKE J. CRAWFORD, MD, Department of Psychological Medicine, Imperial College London, UK; ANNA MARATOS, PGDipMT, Central and North West London Mental Health NHS Trust, UK; ULA NUR, PhD, Imperial College London, UK; ORII McDERMOTT, MMT, Central and North West London Mental Health NHS Trust, UK; SIMON PROCTER, MMT, East London and City NHS Mental Health Trust, UK

Correspondence: Dr Mike Crawford, Department of Psychological Medicine, Claybrook Centre, St Dunstan's Road, London W6 8RP,UK. Tel: +44 (0)207386 I233; fax: +44 (0)207386 I216; email: m.crawford@imperial.ac.uk

(First received 8 July 2005, final revision 24 January 2006, accepted 3 March 2006)

Pavlicevic, M., Trevarthen, C. \& Duncan, J. (1994) Improvisational music therapy and the rehabilitation of persons suffering from chronic schizophrenia. Journal of Music Therapy, 31, 86-104.

Rubin, D. B. (1987) Multiple Imputation for Non-Response in Surveys. New York: John Wiley.

\section{Sainsbury Centre for Mental Health (1998) Acute}

Problems: A Survey of the Quality of Care in Acute Psychiatric Wards. London: Sainsbury Centre for Mental Health

Siris, S. G. (2000) Depression in schizophrenia: perspective in the era of "atypical" antipsychotic agents. American Journal of Psychiatry, I57, 1379-1389.

Tang, W., Yao, X. \& Zheng, Z. (1994) Rehabilitative effect of music therapy for residual schizophrenia. A one-month randomised controlled trial in Shanghai. British Journal of Psychiatry, 165 (suppl. 24), 38-44.

Turry, A. (1998) Transference and counter-transference in Nordoff-Robbins music therapy. In The Dynamics of Music Psychotherapy (ed. K. Bruscia). Gilsum, NH: Barcelona.

van Os, J., Gilvarry, C., Bale, R., et al (1999) To what extent does symptomatic improvement result in better outcome in psychotic illness? UK700 Group. Psychological Medicine, 29, II83-1195.

World Health Organization (1992) Tenth Revision of the International Classification of Diseases and Related Health Problems (ICD-10). Geneva: WHO. 